Website: http://revistas.lamolina.edu.pe/index.php/acu/index

(C) Universidad Nacional Agraria La Molina, Lima - Perú

\title{
Evaluación de niveles de ensilado fijado y seco de vísceras de trucha (Oncorhynchus mykiss) en el crecimiento y engorde de cuyes (Cavia porcellus)
}

\author{
Assessment of levels of fixed and dry silage of trout (Oncorhynchus mykiss) guts in the growth and \\ fattening of guinea pigs (Cavia porcellus)
}

\author{
Sarria Bardales, José A. ${ }^{1 *}$; Barrantes Campos, Cecilio A. ${ }^{2}$; Cantaro Segura, Jose L. ${ }^{2}$ \\ * Autor de correspondencia
}

\section{Resumen}

El estudio evaluó el efecto del insumo denominado ensilado fijado y seco de vísceras de trucha (EFSVT), en dietas de crecimiento y engorde de cuyes (Cavia porcellus); experimento que se realizó en el Laboratorio de Animales Menores de la Universidad Nacional Agraria La Molina, durante los meses de enero y febrero de 2017 por un lapso de siete semanas. Se utilizaron 48 cuyes machos destetados (Tipo 1) de $14 \pm 3$ días de edad, diseñándose tres tratamientos: un testigo (T1, $0 \%$ ) y otros dos con niveles de $3 \%$ (T2) y $6 \%$ (T3) de EFSVT, considerando dieciséis animales por tratamiento, con cuatro repeticiones de cuatro animales cada una. Los alimentos balanceados integrales brindados fueron isoproteícos e isoenergéticos $(20,3 \%$ PT y 2,80 Mcal ED/kg) siendo administrados ad libitum. El peso final fue inferior en el tratamiento $3(\mathrm{p}<0,05)$; sin embargo, en ningún tratamiento hubo significado estadístico en ganancia de peso, conversión alimenticia ni rendimiento de carcasa. El consumo de materia seca, sin diferencia significativa, fue decreciente en relación al incremento del EFSVT en la dieta con 390,9, 378,6 y 350,6 g/día, para T1, T2 y T3, respectivamente. Se concluye que no hubo efecto $(\mathrm{p}<0,05)$ de incluir EFSVT sobre ganancia de peso, consumo, conversión alimenticia y rendimiento de carcasa en el crecimiento y engorde de cuyes. Y dada la tendencia decreciente en el consumo y ganancia de peso cuando se incrementa el porcentaje de EFSVT este insumo puede incluirse hasta un nivel de $3 \%$.

Palabras clave: ensilado de vísceras de trucha; cuy; alimentación; crecimiento y engorde.

\begin{abstract}
The study evaluated the effect of the so-called fixed and dry silage of trout viscera (EFSVT), on growth and fattening diets of guinea pigs (Cavia porcellus); experiment that was carried out in the Laboratory of Minor Animals of the National Agrarian University La Molina, during the months of January and February of 2017 for a lapse of seven weeks. We used 48 male guinea pigs weaned (Type 1) from $14 \pm 3$ days of age, designing three treatments: one control (T1, 0 $\%$ ) and two others with levels of $3 \%$ (T2) and $6 \%$ (T3) of EFSVT, considering sixteen animals per treatment, with four repetitions of four animals each. The integral balanced foods provided were isoproteic and isoenergetic (20,3\% PT and $2,80 \mathrm{Mcal} \mathrm{ED} / \mathrm{kg}$ ) being administered ad libitum. The final weight was lower in treatment $3(\mathrm{p}<0,05)$; however, in no treatment was there statistical significance in weight gain, feed conversion or carcass yield. The dry matter feed intake, without significant difference, was decreasing in relation to the increase of the EFSVT in the diet with 390,9; 378,6 and $350,6 \mathrm{~g} /$ day, for T1, T2 and T3, respectively. It is concluded that there was no effect $(\mathrm{p}<0,05)$ of including EFSVT on weight gain, consumption, feed conversion and carcass yield in the growth and fattening of guinea pigs. And given the decreasing trend in consumption and weight gain when the percentage of EFSVT increases, this input can be included up to a level of $3 \%$.
\end{abstract}

Key words: Cavia porcellus; Oncorhynchus mykiss; silage of trout guts; feed intake.

\section{Introducción}

El gasto debido a la alimentación animal, en especies como el cuy, representa entre 60 y $80 \%$ del costo de producción (Aliaga et al., 2009), siendo la proteína el nutriente de mayor valor; por ello es importante encontrar insumos económicos de buena calidad como son los subproductos y residuos agroindustriales e industriales en general, que tiendan a disminuir el egreso por este rubro. La búsqueda de alternativas alimenticias que permitan reducir costos, para lograr la mayor eficiencia de los sistemas de producción sostenible en el tiempo, es de suma relevancia; siendo así que desde hace algunos años, alrededor del mundo se vienen realizando estudios sobre el aprovechamiento de residuos de pescado, como fuente de proteína para raciones de animales de granja (Berenz, 2002). Una alternativa eficiente para el tratamiento $y$ disposición de los residuos de pescado es la producción de ensilado para la alimentación animal (Ferraz de Arruda et al., 2007). El ensilado surgió como una necesidad de dar valor agregado a las vísceras de pescados que se obtienen

\footnotetext{
${ }^{1}$ Mg.Sc. Profesor Principal del Departamento de Producción Animal, Facultad de Zootecnia, Universidad Nacional Agraria La Molina, Lima, Perú. 
al momento del faenado para su posterior venta; dado que el procesamiento de pescado genera entre 50 y $60 \%$ de residuos del peso bruto de la pesca, los cuales incluyen cabezas, espinazos, aletas, pieles y vísceras propiamente dichas (Calderón et al., 2017). Cabe mencionar que en el Perú la extracción de trucha en el año 2014 fue de 31 401 toneladas métricas (INEI, 2016), de lo que se puede inferir que en dicho año se produjo 15705 tn. de residuos desaprovechados. Sin embargo, estos residuos son altamente perecibles, por lo que implícitamente requieren un adecuado procesamiento y manejo ambiental.

En América Latina, son varios los países que tienen investigaciones sobre recursos pesqueros; sin embargo, sus desperdicios son poco aprovechados, a pesar que su uso podría aportar beneficios en la alimentación de peces, aves y cerdos, entre otras especies, pues constituyen fuentes calórico/proteicas de bajo costo; siendo vehículos de nutrientes indispensables para el desarrollo, crecimiento y reproducción (Gómez et al., 2014). En tal sentido, el estudio y uso de este tipo de nuevos insumos que aportan altos niveles de proteína y energía digestible a menor costo, resulta interesante en recientes explotaciones cuyícolas comerciales, especialmente en la etapa de crecimiento y engorde, donde se procura que los animales producidos para carne se mantengan no más de 90 días de edad en los galpones de crianza (Aliaga et al., 2009). En regiones como la sierra y selva del país, donde se usan preferentemente forrajes (Solórzano y Sarria, 2014), el nutriente más escaso para la alimentación animal es siempre la proteína; mientras que en la costa los concentrados formulados requieren insumos proteicos $\mathrm{y}$ energéticos de buena calidad y disponibilidad, pero con precios competitivos en el mercado (Solórzano y Sarria, 2014).

En consecuencia, se deben probar nuevos insumos en la producción de cuyes, para determinar su eficiencia en la transformación de carne y obtener un mejor rédito económico en la crianza. Mientras tanto la industria de la trucha en la actualidad, tiene un evidente auge en zonas de pobreza y extrema pobreza de nuestro país, generando importante cantidad de residuos, los que debieran ser manejados adecuadamente, teniendo la opción de realizar el ensilado o hidrolizado de vísceras provenientes de su beneficio; insumo que está siendo inicialmente evaluado en la alimentación en pollos de engorde (Garcés et al., 2015) y en cerdos (Calderón et al., 2017).

La mayor utilidad del referido ensilado radica en su empleo en la formulación de raciones de bajo costo y alto valor nutricional (Padilla, 1996). El ensilado de pescado suele ser utilizado en reemplazo de la harina de pescado en la formulación de raciones para aves (Betancourt et al., 2005; Al-Marzooqi et al., 2010), peces (Barreto et al., 2016) e incluso también se ha iniciado su evaluación en cuyes (Mattos et al., 2003); en este último caso referido a ensilado de merluza (Merluccius gayi) entera que, sin especificar el proceso de ensilaje, obtiene resultados satisfactorios. La utilización de residuos de trucha en la elaboración de ensilados estabilizados y su aplicación en la alimentación de cuyes aún no es una práctica común, por lo que merece especial atención. Esta investigación fue realizada con el objeto de determinar el efecto de la inclusión de ensilado fijado y seco de vísceras de truchas (EFSVT), que en el presente trabajo se mezcló con $50 \%$ de subproducto de trigo (SPT) para facilitar su uso en la preparación del balanceados para cuyes en crecimiento y engorde, midiendo pesos y ganancia de peso, consumo de alimento, conversión alimenticia, rendimiento de carcasa y retribución económica.

\section{Materiales y métodos}

El trabajo se realizó en el galpón de cuyes del Laboratorio de Animales Menores del Programa de Investigación y Proyección Social de Animales Menores (PIPSAM) de la Universidad Nacional Agraria La Molina entre los meses de enero a febrero de 2017. Dicha instalación de 20,00 m. de largo; 6,00 m. de ancho y 3,00 m. de altura, es de material noble con ventanas de malla y cortinas cortaviento de polipropileno, dentro de la cual se encontraban las cuyeras que eran jaulas de alambre electro soldado (1,5 m x $0,8 \mathrm{~m}$ x 0,45 m de altura). Se usaron 48 cuyes machos mejorados recién destetados $(14 \pm 3$ días de edad) del genotipo Cieneguilla - La Molina; los cuales fueron distribuidos aleatoriamente en cada uno de los tres tratamientos, con cuatro repeticiones de cuatro animales en cada unidad experimental (jaula); estadísticamente se planteó un diseño de bloques completamente al azar (SAS, 2013) mientras que la comparación entre medias de tratamientos se hizo con la prueba de rango múltiple de Duncan (Calzada, 1982).

Las dietas evaluaron tres niveles del insumo EFSVT; que tenían 0,3 y $6 \%$ de participación en el balanceado, según se presenta en la Tabla 1. Los balanceados fueron formulados con el programa Mixit-2 a mínimo costo; y las cantidades fueron determinadas por los requerimientos nutritivos mínimos de la especie (Tabla 2). Las vísceras (contenido digestivo y branquias) fueron molidas utilizando una moledora con la criba habitual para carne de $3,5 \mathrm{~mm}$. de diámetro, luego de lo cual se añadió una solución de ácido fórmico (tipo comercial al $85 \%$; en una concentración $2 \mathrm{~N}$ ), con el objeto de lograr la fijación inmediata para su mayor conservación, mezclando esta solución con las vísceras en una proporción de $4 \%$. Posteriormente se colocó la mezcla en un bidón de plástico cerrado herméticamente, por un lapso de tres a cuatro días para obtener el ensilado líquido, el cual es mezclado en una proporción 1:1 con afrecho de trigo hasta obtener la mezcla seca y sobretodo disgregable; para terminar, dicha mezcla fue colocada en un secador solar por espacio de cuatro a cinco días, obteniéndose el insumo denominado "ensilado fijado y seco de vísceras de trucha - EFSVT" (Venturi, 2017), con la composición nutritiva de la Tabla 3 .

Las dietas de alimento integral, preparadas para el sistema de exclusión de forraje, fueron elaboradas cada siete días y suministradas diariamente a las 9 am, garantizando la condición ad libitum. Para el aporte de 
balanceados y agua se usaron pocillos de arcilla. La recolección de datos de consumo fue también diaria a las 8 am. Los animales fueron pesados semanalmente a la misma hora y antes del suministro del alimento. Los pesos, consumo de alimento y conversión alimenticia se tomaron por semana y acumulado, con una balanza analítica (marca KERN) de $2 \mathrm{~kg}$ de capacidad y 0,1 gramos de sensibilidad. La determinación de la materia seca del EFSVT fue por el método AOAC (2005). 950,46. La ganancia de peso se determinó restando el peso vivo final menos el peso vivo inicial. Para el consumo de alimento se midió el consumo promedio por cuy en cada repetición dentro de cada tratamiento. Esta medición, fue el resultado de la diferencia del peso del alimento proporcionado en el día, menos el residuo de alimento dejado por el cuy al día siguiente. En el caso de consumo semanal promedio por animal en materia seca, se dividió el consumo de alimento en materia seca semanal, entre el número de cuyes de cada jaula. La conversión alimenticia se calculó en base al consumo de materia seca de las dietas experimentales sobre la ganancia de peso.

Tabla 1. Dietas formuladas con tres niveles de EFSVT en la alimentación de cuyes

\begin{tabular}{lccc}
\hline Ingredientes & $\mathrm{T} 1$ & $\mathrm{~T} 2$ & $\mathrm{~T} 3$ \\
\hline Subproducto de trigo & 61,5 & 61,5 & 61,5 \\
Hominy feed & 13,0 & 10,8 & 8,5 \\
Torta de soya & 18,0 & 17,3 & 16,5 \\
Heno de alfalfa & 5,0 & 5,0 & 5,0 \\
Carbonato de Calcio & 2,0 & 2,0 & 2,0 \\
DL-Metionina & 0,1 & 0,1 & 0,1 \\
Sal común & 0,1 & 0,1 & 0,1 \\
Vitamina C protegida & 0,2 & 0,2 & 0,2 \\
Premezcla cuy & 0,1 & 0,1 & 0,1 \\
EFSVT & 0,0 & 3,0 & 6,0 \\
Total & 100,0 & 100,0 & 100,0 \\
Precio S/.por kg ración $(*)$ & 1,14 & 1,12 & 1,10 \\
\hline EFSVT: Enil
\end{tabular}

EFSVT: Ensilado fijado y seco de vísceras de trucha (con afrecho de trigo $1: 1)$.

(*) Precio estimado por el Programa de Animales Menores de la UNALM (PIPSAM).

El rendimiento de carcasa fue evaluado midiendo la relación del peso de la canal con respecto al peso vivo antes del beneficio del cuy; para este efecto los animales fueron dejados en ayuno previo por 24 horas. La carcasa estuvo conformada por piel, cabeza, tronco, abdomen, miembros anteriores, miembros posteriores y vísceras nobles (corazón, pulmón, hígado y riñones). Se hizo análisis de varianza para evaluar la ganancia de peso, el consumo de alimento y la conversión alimenticia. Para el rendimiento de la carcasa, de cada repetición se escogió al azar dos cuyes por tratamiento, con lo cual se configuró un modelo anidado. La retribución económica relativa (RER) se determinó por diferencia entre el precio de la carcasa y el costo de alimentación en cada tratamiento.
Tabla 2. Composición nutritiva de las dietas experimentales para cuyes en base húmeda (BH) y en base seca (BS)

\begin{tabular}{lcccccc}
\hline \multirow{2}{*}{ Nutrientes } & \multicolumn{2}{c}{ T1 } & \multicolumn{2}{c}{ T2 } & \multicolumn{2}{c}{ T3 } \\
& BH & BS & BH & BS & BH & BS \\
\hline Materia Seca & 89,4 & 100,0 & 89,8 & 100,0 & 89,6 & 100,0 \\
Humedad & 10,6 & 0,0 & 10,2 & 0,0 & 10,4 & 0,0 \\
Grasa & 4,8 & 5,3 & 4,9 & 5,4 & 5,1 & 5,7 \\
Cenizas & 6,2 & 6,9 & 6,3 & 7,1 & 6,4 & 7,1 \\
Proteína Bruta & 19,9 & 22,3 & 20,7 & 23,1 & 20,3 & 22,7 \\
ELN & 49,6 & 55,4 & 49,7 & 55,3 & 49,8 & 55,6 \\
ED (Mcal/Kg)** & 2,8 & 3,2 & 2,8 & 3,1 & 2,8 & 3,1 \\
\hline
\end{tabular}

Fuente: Laboratorio de Evaluación Nutricional de Alimentos del Departamento de Nutrición Animal de la Universidad Nacional Agraria La Molina (LENA-UNALM).

(**) Valor estimado (Robalino, 2008).

Tabla 3. Composición nutritiva de restos de trucha y de EFSVT

\begin{tabular}{lcc}
\hline Nutrientes (\%) & Restos de Trucha & EFSVT \\
\hline Proteína & 32,11 & 22,05 \\
Fibra & 0,00 & 8,15 \\
Extracto etéreo & 63,68 & 18,91 \\
Extracto libre de nitrógeno & 0,00 & 43,40 \\
Cenizas & 4,21 & 7,50 \\
Materia seca & 100,00 & 100,00 \\
\hline
\end{tabular}

Fuente: LENA - UNALM.

\section{Resultados y discusión}

\section{Pesos y ganancias de peso}

La Tabla 4 muestra resultados estadísticamente iguales en ganancia de peso promedio y acumulado para los tres tratamientos $(p>0,05)$. Sin embargo, en cuanto a pesos finales se observó diferencias significativas $(p<0,05)$, obteniendo T1 y T2 pesos finales superiores al T3 (6\% de EFSVT) que solo llegó a 904,2 g. Los resultados obtenidos son algo inferiores a los encontrados por Condori (2014) en similar lapso de evaluación, quien reporta valores entre 1043 y 1062 g. a los 63 días de evaluación en dietas de inicio y crecimiento de cuyes con exclusión de forraje y bajos niveles de fibra. Por otra parte, al comparar numéricamente las semanas 4 y 6 de la evaluación, el T2 revela mejor velocidad de crecimiento en relación a los otros dos tratamientos. Además, en la sexta semana de evaluación, los cuyes del mismo T2 pesaban en promedio 917,9 g. de peso vivo, lo cual indica que ya estaban listos para ser comercializados; evidenciando que la inclusión de ensilado de trucha al $3 \%$, podría ser un valioso insumo en la etapa de engorde de los cuyes. Los datos permiten evidenciar que los tratamientos que se comportaron ligeramente mejor fueron el T1 y T2; la aceptable ganancia de peso en dietas que contienen ensilado de pescado, se pueden direccionar a su pre digestión proteica; ya que los aminoácidos se hacen más absorbibles (Berenz, 1998). Estos resultados son parecidos a lo obtenido por Mattos et al. (2003), donde evaluando el uso de ensilado biológico de pescado (merluza) sin adición de afrecho en la alimentación 
de cuyes, se mostró mejor ganancia de peso vivo con dietas que contenían $30 \%$ de ensilado del pescado entero; no obstante es probable que la palatabilidad del alimento se viera afectada en este caso, originándose menor consumo de alimento.

\section{Consumo de alimento}

La misma Tabla 4, muestra adicionalmente resultados estadísticamente iguales en el consumo de alimento en acumulada $(\mathrm{p}>0,05)$ de los tres tratamientos. Los resultados obtenidos de conversión alimenticia acumulada denotan menor eficiencia a lo reportado por Condori (2014) quien logra conversiones entre 2,79 y 3,04. Sin embargo, al comparar numéricamente la quinta semana, el T2 y T3 muestran resultados similares entre sí, pero mejores al T1. Es decir, la transformación de alimento en peso vivo al inicio de la etapa de acabado es mejor en estos tratamientos que en el control.

Tabla 4. Peso (g), ganancia de peso (g), consumo de materia seca (g/día) y conversión alimenticia en cuyes alimentados con dietas conteniendo tres niveles de ensilado de pescado

\begin{tabular}{|c|c|c|c|c|c|c|c|c|c|c|c|}
\hline \multirow{2}{*}{ Semana } & \multirow[b]{2}{*}{ PV } & \multicolumn{3}{|c|}{ T1 (0\%) } & \multicolumn{3}{|c|}{$\mathrm{T} 2(3 \%)$} & \multirow[b]{2}{*}{ PV } & \multicolumn{3}{|c|}{ T3 $(6 \%)$} \\
\hline & & GDP & CMS & CA PV & GDP & CMS & $\mathrm{CA}$ & & GDP & CMS & $\mathrm{CA}$ \\
\hline 0 & $291,9^{\mathrm{a}}$ & --- & --- & $---300,6^{\mathrm{a}}$ & --- & --- & --- & $294,0^{\mathrm{a}}$ & --- & --- & --- \\
\hline 1 & 382,8 & 90,9 & 224,7 & $2,5388,3$ & 87,7 & 220,5 & 2,6 & 367,1 & 73,1 & 214,6 & 2,9 \\
\hline 2 & 481,5 & 98,7 & 310,4 & $3,2479,9$ & 91,6 & 298,0 & 3,3 & 447,5 & 80,4 & 272,8 & 3,5 \\
\hline 3 & 589,6 & 108,1 & 363,0 & $3,4578,3$ & 98,4 & 346,8 & 3,5 & 524,1 & 82,9 & 313,9 & 3,9 \\
\hline 4 & 698,9 & 109,3 & 427,1 & $3,9697,1$ & 118,9 & 406,4 & 3,5 & 608,3 & 90,4 & 351,2 & 4,0 \\
\hline 5 & 782,9 & 83,9 & 416,9 & $5,1791,6$ & 94,5 & 380,8 & 4,1 & 714,7 & 93,9 & 346,0 & 3,7 \\
\hline 6 & 889,8 & 106,9 & 489,7 & 4,7 917,9 & 126,1 & 493,0 & 3,9 & 823,9 & 109,3 & 470,4 & 4,3 \\
\hline 7 & $968,4^{\mathrm{a}}$ & 78,7 & 504,1 & $6,7968,9^{\mathrm{a}}$ & 51,1 & 504,5 & 10,5 & $904,2^{\mathrm{b}}$ & 80,3 & 485,7 & 6,5 \\
\hline Promedio & & $96,7^{\mathrm{a}}$ & $390,8^{\mathrm{a}}$ & $4,2^{\mathrm{a}}$ & $95,5^{\mathrm{a}}$ & $378,6^{\mathrm{a}}$ & $4,5^{\mathrm{a}}$ & & $87,2^{\mathrm{a}}$ & $350,6^{\mathrm{a}}$ & $4,1^{\mathrm{a}}$ \\
\hline Acumulado & --- & $676,6^{\mathrm{a}}$ & $2,735,9^{\mathrm{a}}$ & ${ }^{a} 4,1^{a} \quad---$ & $668,3^{\mathrm{a}}$ & $2,650,1^{\mathrm{a}}$ & $4,0^{\mathrm{a}}$ & --- & $610,2^{a}$ & $2,454,4^{\mathrm{a}}$ & $4,0^{\mathrm{a}}$ \\
\hline
\end{tabular}

materia seca promedio y acumulada de los tres tratamientos. Los valores de consumo total de alimento son similares a lo encontrado por Condori (2014) quien reporta entre 2495 y 2639 g.; sin embargo, el consumo de alimento por día del experimento -entre 50,08 y 55,83 g.- es algo superior a lo encontrado por el referido autor, quien obtiene valores de 45,32 a 48,95 g/d. Por otro lado, cuando se compara numéricamente los promedios de consumos acumulados, se observa una gradiente inversa al nivel de inclusión del insumo probado; siendo así que el T3 muestra menor consumo de alimento que los otros dos tratamientos. Desde un punto de vista económico, esto podría ser considerado bueno, ya que el animal consume menos alimento para una ganancia de peso vivo similar a los demás tratamientos. Pero, como indican Mattos et al. (2003), ello puede deberse a la menor palatabilidad de alimento cuanto mayor es la inclusión de ensilado de pescado. Por otro lado, también se observa numéricamente que en las semanas 3, 4 y 5 de evaluación, el T3 muestra similares consumos al T2 pero inferior al T1 (control); no obstante en las siguientes semanas, el consumo se niveló en los tres tratamientos. Lo que indica que los cuyes que recibieron EFSVT, en su etapa de crecimiento consumían menos alimento, mientras que en la etapa final el consumo se igualó; ello induce a suponer que probablemente el insumo evaluado podría ser considerado mejor alternativa en dietas de acabado de cuyes, que en inicio o crecimiento.

\section{Conversión alimenticia}

La Tabla 4, también nos muestra resultados similares en la conversión alimenticia en materia seca promedio y
Mientras que, en la séptima semana de evaluación, el T3 y T1, presentan similares conversiones alimenticias, pero superiores al T2. En consecuencia, para la etapa de acabado, la inclusión del ensilado de víscera de trucha, puede dar mejores índices de conversión alimenticia en los cuyes. Similares resultados son indicados por Mattos et al. (2003), que con inclusiones de $20 \%$ de ensilado biológico de pescado (merluza entera) en cuyes, obtienen mejor conversión alimenticia.

\section{$\underline{\text { Rendimiento de carcasa }}$}

En la Tabla 5, se presenta resultados estadísticamente similares en rendimiento de carcasa, con previo ayuno de 24 horas, para los tres tratamientos $(p>0,05)$. Los valores obtenidos para este indicador, son superiores a lo encontrado por Condori (2014) que determina rendimientos que varían entre 67,07 y $69,51 \%$. Por los resultados logrados, se infiere que la inclusión del ensilado de víscera de trucha no tiene efecto en el rendimiento carnicero de los cuyes, pudiendo ser usado como un insumo para el engorde de esta especie. Cabe indicar, que en el efecto anidado o de medidas repetidas, los cuyes escogidos al azar de cada jaula de evaluación respondieron de manera similar $(\mathrm{p}>0,05)$, deduciendo que este valor sería similar a todos los cuyes de la evaluación. En contraste, Mattos et al., (2003), indican que la inclusión de $30 \%$ de ensilado de pescado, muestra mejor rendimiento de canal que los demás tratamientos, lo cual puede deberse a que usó diez veces más del insumo de pescado y en forma entera, con respecto a la presente investigación. 
Tabla 5. Efecto de dietas con diferentes niveles de EFSVT sobre el rendimiento de carcasa (\%)

\begin{tabular}{cccc}
\hline Jaula & Tratamiento & T3 \\
& 73,85 & 75,75 & 69,45 \\
2 & 73,80 & 74,40 & 74,90 \\
3 & 74,25 & 75,60 & 74,20 \\
4 & 78,65 & 75,10 & 73,00 \\
\hline Promedios & $75,14^{\mathrm{a}}$ & $75,21^{\mathrm{a}}$ & $72,89^{\mathrm{a}}$ \\
\hline
\end{tabular}

Retribución y mérito económico

Los resultados de la retribución económica relativa (RER) y el mérito económico se muestran en la Tabla 6. El mejor mérito económico se observó en T2 (3\% de EFSVT) con un ligero $1,14 \%$ superior al control, explicándose ello por similares ganancias de peso frente a menores costos, debido al menor consumo de alimento. Mientras que con el T3 (6 \% de EFSVT), se obtiene 5,09 \% menos retribución que con el testigo.

Tabla 6. Retribución económica y mérito económico de cuyes alimentados con dietas conteniendo tres niveles de EFSVT

\begin{tabular}{lccc}
\hline Índice & $\mathrm{T} 1$ & $\mathrm{~T} 2$ & $\mathrm{~T} 3$ \\
Peso final cuy (kg) & 0,968 & 0,969 & 0,904 \\
Precio cuy (S/./cuy) & 18,00 & 18,00 & 18,00 \\
$\begin{array}{l}\text { Ingreso bruto por kg. de cuy logrado (S/.) } \\
\text { Alimento balanceado (dieta) }\end{array}$ & $2,74,432$ & 17,440 & 16,275 \\
Consumo de alimento (kg) & 2,65 & 2,45 \\
Costo del alimento (S/. kg) & 1,14 & 1,12 & 1,10 \\
Total Egreso por alimento (S/.) & 3,124 & 2,968 & 2,695 \\
$\begin{array}{l}\text { Retribución económica relativa por kg. de } \\
\text { cuy logrado. }\end{array}$ & 14,31 & 14,47 & 13,58 \\
\hline Mérito económico (\%) & 100,00 & 101,14 & 94,91 \\
\hline
\end{tabular}

\section{Conclusiones}

El ensilado fijado y seco de vísceras de trucha (EFSVT) incluido, en la presentación y las proporciones empleadas en el presente experimento, en los balanceados de los cuyes en la etapa de crecimiento y engorde no tiene influencia estadística en ganancia de peso, consumo acumulado, conversión alimenticia ni en el rendimiento de carcasa de los animales.

El EFSVT puede incorporarse en dietas de cuyes hasta un nivel de $3 \%$, dado que muestra la mejor retribución económica y la tendencia de los resultados a mostrar ganancias de peso y consumo de alimento decrecientes cuando aumenta el porcentaje de EFSTV en la dieta.

\section{Recomendaciones.}

Continuar la realización de pruebas de este tipo, a fin de afinar la inclusión de este insumo en la alimentación de los cuyes en diferentes niveles y presentaciones; así como en distintas etapas productivas, como es la reproducción de los cuyes.
Promover estudios y procesos para la homogenización y estandarización del ensilado fijado y seco de vísceras de trucha (EFSVT), como insumo para la alimentación animal.

Estimar la digestibilidad del ensilado de vísceras de trucha en diferentes especies pecuarias, para el cálculo real de su contenido energético y aporte nutricional.

- Desarrollar pruebas de degustación a fin de tener comparaciones sensoriales con diferentes niveles de inclusión, en distintas especies.

\section{Agradecimientos}

Se agradece a la Empresa "Grupo Wilkay S.A.C" de Víctor Venturi y al Proyecto "Innóvate Perú", a Juancarlos Cruz Luís, Alexandra Mosqueira Robles y Daniel Huamaní Bedoya por el apoyo técnico para esta investigación.

\section{Literatura citada}

AOAC [Association of official analytical chemist]. 2005. Official methods of analysis. 13th Edition. Arlington USA.

Aliaga, L.; Moncayo, R.; Rico, E. y Caycedo, A. 2009. Producción de cuyes. Fondo Editorial UCSS. Universidad Científica Sedes Sapientiae. Lima - Perú. $808 \mathrm{p}$.

Al-Marzooqi, W.; Al-Farsi, A.; Kadim, T.; Mahgoub, O. and Goddard, S. 2010. The effect of feeding different levels of sardine fish silage on broiler performance. Meat quality and sensory characteristics under closed and open-sided housing systems. Asian Australs Journal Animal Science, 23: 1614-1625. DOI: 10.5713/ ajas.2010.10119

Barreto, F.; Parés, G.; Correa, G.; Durazo, E. and Viana, T. 2016. Total and partial fishmeal substitution by poultry byproduct meal (pet food grade) and enrichment with acid fish silage in aquafeeds for juveniles of rainbow trout Oncorhynchus mykiss. Lat Am J Aquat Res., 44: 327-335. DOI: 10.3856/vol44- issue2-fulltext-13.

Berenz, Z. 1998. Ensilado de residuos de pescado. En: Procesamiento de Ensilado de Pescado. XIV Curso Internacional de Tecnología de Procesamiento de Productos Pesqueros. 7 de Enero - 27 de Febrero. ITPJICA. Callao. 18-70p.

Berenz, Z. 2002. Utilización de residuos de pescado en pollos. Callao (Perú): Instituto Tecnológico Pesquero del Perú. 32-34p.

Betancourt, L.; Díaz, J.; Aguilar, X. y Ríos, J. 2005. Efecto del ensilaje de vísceras de trucha (Oncorhynchus mykiss) sobre el comportamiento productivo y el contenido de ácidos omega-3 en hígado, muslos y pechuga de pollos de engorde. Livestock Research for Rural Development. Volume 17, Article \#106.

Disponible en: http://www.lrrd.org/lrrd17/9/beta17106. htm 
Calzada J. 1982. Métodos estadísticospara la investigación. Lima: Ed Jurídica. 644p.

Calderón, V.; Churacutipa, M.; Salas, A.; Barriga, M. y Aranibar, M. 2017. Inclusión de ensilado de residuos de trucha en el alimento de cerdos y su efecto en el rendimiento productivo y sabor de la carne. Revista de Investigaciones Veterinarias del Perú, 28(2): 265-274. DOI: $10.15381 /$ rivep.v28i2.13055.

Condori, R. 2014. Evaluación de bajos niveles de fibra en dietas de inicio y crecimiento de cuyes (Cavia porcellus) con exclusión de forraje. Tesis de Ingeniero Zootecnista. Universidad Nacional Agraria La Molina, Lima, Perú.77 p.

Ferraz de Arruda, L.; Borghesi, R. and Oetterer M. 2007. Use of fish waste as silage - a review. Braz Arch Biology Technology, 50: 879-886. DOI: 10.1590/ S1516- 89132007000500016.

Garcés, Y.; Pereal, C.; Valencia, N.; Hoyos, J. y Gómez, A. 2015. Efecto nutricional del ensilado químico de subproductos piscícolas en la alimentación de pollos de engorde (Gallus domesticus et al.). Cuban Journal of Agricultural Science, 49(4):503-508.

Gómez, G.; Ortiz, M.; Perea, C. and López, F. 2014. Evaluación del ensilaje de vísceras de tilapia roja (Oreochromis spp) en alimentación de pollos de engorde. Biotecnología en el Sector Agropecuario y Agroindustrial, 12(1): 106-114.

INEI [Instituto Nacional de Estadística e Informática]. 2016. Series nacionales. Extracción de trucha fresca. [Internet]. Disponible en: http://series.inei.gob.pe:8080/ sirtod-series/

Mattos, J.; Chauca, L.; San Martin, F.; Carcelén, F. y Arbaiza, T. 2003. Uso del ensilado biológico de pescado en la alimentación de cuyes mejorados. Revista de Investigaciones Veterinarias del Perú, 14(2):89 -96.

Padilla, P. 1996. Técnicas del ensilado biológico de residuos de pescado para la ración animal. Nota técnica. Instituto de Investigaciones de la Amazonia Peruana IIAP. Iquitos, Folia Amazónica No 8, 147 - 151p.

Robalino, P. 2008. Valoración energética de diferentes tipos de harina de pescado, torta de palmiste y torta de algodón utilizadas en la alimentación de cuyes (Cavia porcellus). Tesis de grado para la obtención del título de Ingeniero Zootecnista. Escuela Superior Politécnica de Chimborazo. Riobamba - Ecuador. 35p.

SAS [Statistical Analysis System]. 2013. User's Guide (Release 9.4). Cary, North Carolina, USA: SAS Institute.

Solórzano, J. y Sarria, J. 2014. Crianza.; producción y comercialización de cuyes. Colección Agro. Editorial Macro. Lima - Perú. 194 p.

Venturi, V. 2017. Entrevista personal; Líder de Proyecto Fincyt. "Elaboración de ensilado de víscera de truchas como un insumo alimenticio". Lima, Perú. 